Research Article

\title{
Bentonite-Based Nano Organic Clay Using Chalcone and Azo Dye as Organophilic Reagents
}

\author{
Manar Ghyath Abd-Almutalib Al-Mosawy ${ }^{\mp}$, Emad Abbas Jaffar Al-Mulla ${ }^{\circledR}$, Majed Jari Mohamad \\ Department of Chemistry, Faculty of Science, University of Kufa, P.O. Box 21, An-Najaf 54001, Iraq. \\ —Corresponding authors. E-mail: manarghyth@yahoo.com; imad.almulla@uokufa.edu.iq
}

Received: Apr. 25, 2017; Accepted: May 26, 2017; Published: Jun. 5, 2017.

Citation: Manar Ghyath Abd-Almutalib Al-Mosawy, Emad Abbas Jaffar Al-Mulla, and Majed Jari Mohamad, Bentonite-Based Nano Organic Clay Using Chalcone and Azo Dye as Organophilic Reagents. Nano Biomed. Eng., 2017, 9(2): 124-1 28.

DOI: $10.5101 /$ nbe.v9i2.p124-128.

\begin{abstract}
Organclays (OBNTs) were prepared using two new organic surfactants including 3-(4-aminophenyl)1-(4-chlorophenyl)prop-2-en-1-on (CH) synthesized from a Claisen-Schmidt condensation reaction between p-aminoacetophenone and p-chlorobenzaldehyde; and 4-((4-nitrophenyl)diazenyl)-N(pyrimidin-2-yl) (AZ) prepared from reaction of sulfadiazine and p-nitoaniline. Organclays OBNTs were characterized by Fourier transform infrared spectroscopy (FTIR) to evaluate the incorporation of surfactants in bentonite. The X-ray diffraction (XRD) technique was utilized to indicate the basal spacing of the treated clay as a measure of susceptibility of new organoclays. The FTIR and XRD results showed that the OBNTs were successfully incorporated in the layers of bentonite clay. Thermogravimetric analysis (TGA) was also used to determine thermal stability. An enhancement in stability was observed in OBNTs compare to pure bentonite. OBNTs prepared in this study can be used to produce disposable packaging a promising polymer nanocomposite.
\end{abstract}

Keywords: Sodium bentonite; Modification; Surfactants; Organic cations

\section{Introduction}

Bentonite, which is predominantly amontmorillonitic clay, is characterized by one Al octahedral sheet placed between two Si tetrahedral sheets. The isomorphous substitution of $\mathrm{Al}^{3+}$ for $\mathrm{Si}^{4+}$ in the tetrahedral layer and $\mathrm{Mg}^{2+}$ for $\mathrm{Al}^{3+}$ in the octahedral layer results in a net negative surface charge on the clay. This charge imbalance is offset by exchangeable cations typically $\mathrm{Na}^{+}$and $\mathrm{Ca}^{2+}$ at the clay surface. The layered structure of the clay allows expansion after wetting. $\mathrm{Na}^{+}$and $\mathrm{Ca}^{2+}$ are strongly hydrate in the presence of water, resulting in ahydrophilic environment at the clay surface [1].
However, cation-exchange reaction have been traditionally exploited as an effective method to replace these inorganic ions with organic cationic surfactant molecules, which intercalate into the clay gallery, resulting in expansion of the interlayer spacing and leading to an increase in the basal spacing. These organic cations render the surface of the clay mineral hydrophobic, leading to the increase of the clay wetttability and providing favourable interactions with organic molecules. Both organic-modified and unmodified clays have been used for different industrial applications such as rheological additives, thickeners in coating products glues, plastisols, drilling fluids and cosmetics [2, 3]. 
Recently, clays were used in the field of materials science such as solid phase polymeric nanocomposites. In the 1990's, the use of organically modified clays in the polymer-clay nanocomposites has attracted researchers into this area of materials science and technology [4].

The organic modification of clay minerals leads to a decrease in surface energy making clays compatible with polymers. The surface energy of clay minerals and polymer can be determined from contact angle measurements [5]. Melt processed nylon 6-clay nanocomposites have been prepared using organoclay [6]. It was reported that, based on $\mathrm{X}$-ray diffraction analysis, various arrangement of alkyl chains in organoclays had proposed. Novel organomontmorillonites have been synthesized and characterized using different ammonium compounds [7-8]. In this study, two new different ammonium compounds 3-(4-aminophenyl)-1-(4-chlorophenyl) prop-2-en-1-on (CH) and 4-((4-nitrophenyl)diazenyl)$\mathrm{N}$-(pyrimidin-2- yl)(AZ) were used to modify the compatibility of bentonite clay. These organoclays can be used in both medical and industrial applications specially in both promising polymer and biopolymer nanocomposites.

\section{Experimental Material}

Hydrochloric acid from J. T. Baker, USA. Sodium hydroxide was obtain from merck, British. p-aminoacetophenone and p-chlorobenzaldehyde were obtained from Fluka, Germany.

\section{Characterization \\ XRD analysis}

The X-ray diffraction (XRD) study was carried out using a Shimadzu XRD 6000 diffractometer with $\mathrm{Cu} \mathrm{K}$ radiation $(\mathrm{k}=0.15406 \mathrm{~nm})$. The diffractogram was scanned in the ranges from 2 to $10^{\circ}$ at a scan rate of $1 \%$ min.

\section{FTIR spectroscopy}

The fourier transform infrared spectroscopy (FTIR) spectra of the blends samples were recorded by the FTIR spectrophotometer (Perkin Elmer FTIRSpectrum BX, USA) using $\mathrm{KBr}$ disc technique.

\section{NMR techniques}

${ }^{1} \mathrm{H}$-NMR (nuclear magnetic resonance) and

${ }^{13} \mathrm{C}-\mathrm{NMR}$ spectra were obtained with Bruker,
Ultra Shield $300 \mathrm{MHz}$ and $75 \mathrm{MHz}$, using dimethyl sulfoxide (DMSO) as solvent and transcranial magnetic stimulation (TMS) as an interanal standard.

\section{TGA analysis}

Thermogravimetric analysis (TGA) was recorded by thermal gravimetric analyzer, STA PT-1000 linseis TGA, Germany.

\section{Preparation of chalcone [9]}

$0.5 \mathrm{~g}$ (0.002 mol) p-aminoacetophenone and $1 \mathrm{mmol}$ p-chlorobenzaldehyde, were mixed with aqueous solution $10 \% \mathrm{NaOH}$. $0.5 \mathrm{~mL}$ of $99 \% \mathrm{EtOH}$ was then added to the mixture. The resulting mixture was stirred at room temperature for $4 \mathrm{hrs}$, and then poured in to ice water. After that, $10 \% \mathrm{HCl}$ solution was added to the product. The solid formed was filtered then washed with ethanol and water at a ratio of 10:5. Recrystallization from 99\% ethanol afforded produced pure chalcone (67\% yield, yellow solid, m.p. $160-165^{\circ} \mathrm{C}$ ).

\section{Preparation of azo [10]}

$2 \mathrm{gm}(0.0145 \mathrm{~mol})$ of 4-nitro aniline was dissolved in $3 \mathrm{~mL}$ of concentrated hydrochloric acid and $15 \mathrm{~mL}$ of distilled water. The mixture was cooled at $0-5{ }^{\circ} \mathrm{C}$ in icewater bath, then $0.9 \mathrm{~g}$ ( 0.0145 mole) of a solution of sodium nitrite was dissolved in $5 \mathrm{~mL}$ of distilled water and then cooled at $0-5{ }^{\circ} \mathrm{C}$. This solution was added a drop wise to the mixture with stirring at the same temperature. Design amount of diazonum salt solution was added portion wise to solution of $3.62 \mathrm{~g}(0.0145$ mol) sulfadiazine in distilled water with $1.5 \mathrm{~g}$ of sodium hydroxide dissolved in $100 \mathrm{~mL}$ distilled water. The $\mathrm{pH}$ was maintained with 6-7 and temperature at $0-5{ }^{\circ} \mathrm{C}$. The mixture was stirred for $30 \mathrm{~min}$ and then left over night. The product was precipitated and filtered, washed well with sodium bicarbonate and distilled water undercrystallized from ethanol (yield 72\%, brown solid, m.p. 193-198 ${ }^{\circ} \mathrm{C}$ ).

\section{Preparation of organoclays (OBNT) [11]}

Sodium Bentonite (Na-BNT) $4.00 \mathrm{~g}$ was stirred vigorously in $600 \mathrm{~mL}$ of hot distilled water for $1 \mathrm{~h}$ to form a clay suspension. Subsequently, $4.50 \mathrm{~g}$ of both $\mathrm{CH}$ and $\mathrm{AZ}$, which had been dissolved in $400 \mathrm{~mL}$ of hot water and $16.00 \mathrm{~mL}$ concentrated hydrochloric acid was added into the clay suspension. After being stirred vigorously for $1 \mathrm{~h}$ at $80^{\circ} \mathrm{C}$, the organoclay suspension was filtered and washed with distilled water until no chloride was detected with a $1.0 \mathrm{~mol}$ silver nitrate solution. It was then dried at $60{ }^{\circ} \mathrm{C}$ for $72 \mathrm{~h}$. The dried organoclay was ground until the particle size was $100 \mu \mathrm{m}$ [12-13]. Scheme 1 shows the structures of $\mathrm{CH}$ and AZ 


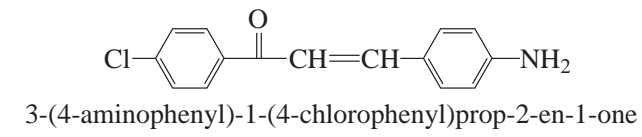

(a)

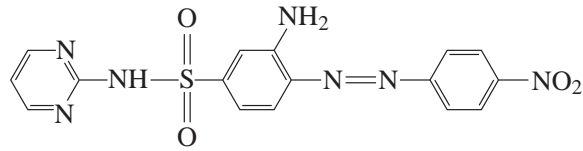

3-amino-4-((4-nitrophenyl)diazenyl)- $N$-(pyrimidin-2-yl)benzenesulfonamide

(b)

Scheme 1 The structures of (a) $\mathrm{CH}$; and (b) AZ.

used to prepare organoclays.

\section{Results and Discussion XRD analysis}

The alkyl ammonium cation exchange enables the conversion of the hydrophilic interior clay surface into the hydrophobic surface and consequently increase the layer distance aswell [14]. In this study, Na-BNT was surface treated with $\mathrm{CH}$ and $\mathrm{AZ}$ as intercalation agent through cation exchange process. The cationic head groups of the intercalation agent molecule would preferentially reside at the layer surface and the tail of the compound will radiate a ways from the surface. The presence of these chains in the galleries makes the originally hydrophilic silicate to organophilic and thus increase the layer-to-layer spacing of $\mathrm{Na}-$ BNT [15]. The organoclays prepared in this work, 3-(4-aminophenyl)-1-(4-chlorophenyl)prop-2-en-1onBNT (CH-BNT) and 4-((4-nitrophenyl)diazenyl)-N(pyrimidin-2-yl)-BNT (AZ-BNT), were investigated using XRD measurements in the $2 \Theta$ ranges from $2-10^{\circ}$. Na-BNT shows diffraction peak at $2 \Theta=7.0762$ which assigns to the interlayer distance of the natural bentonite with a basal spacing of $1.25 \mathrm{~nm}$ [16]. After the ion exchange reaction, the basal spacing (d001 value) increases from 1.25 (Na-BNT) to 1.76 and 1.77 for CH-BNT and AZ-BNT, respectively (Fig. 1).

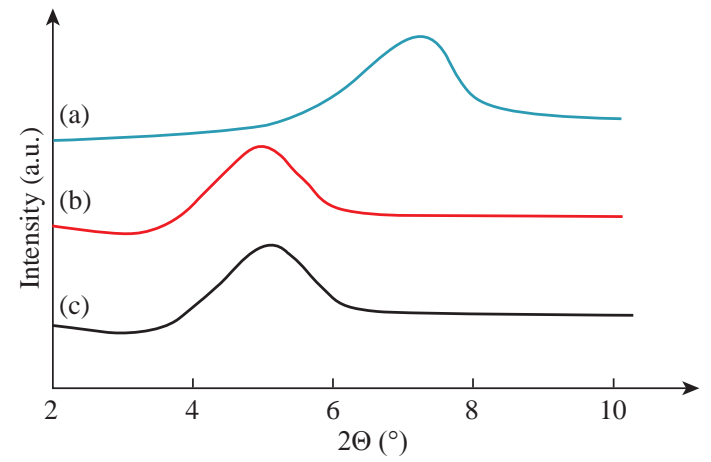

Fig. 1 The XRD patterns of (a) Na-BNT; (b) CH-BNT; and (c) AZ-BNT.
Table 1 Diffraction angle and basal spacing of bebtonite and its modification with different organic cations

\begin{tabular}{lccc}
\hline Sample & Exchanged cation & $2 \Theta\left(^{\circ}\right)$ & $\mathrm{d}(001)$ spacing $(\mathrm{nm})$ \\
\hline Bentonite & $\mathrm{Na}^{+}$ & 7.08 & 1.25 \\
$\mathrm{CH}$ & $\mathrm{C}_{15} \mathrm{H}_{9} \mathrm{ClO} \mathrm{NH}{ }^{+} \mathrm{CH}^{+}$ & 4.99 & 1.77 \\
$\mathrm{AZ}$ & $\mathrm{C}_{16} \mathrm{H}_{9} \mathrm{~N}_{5} \mathrm{O}_{4} \mathrm{SNH}_{3}^{+} \mathrm{AZ}^{+}$ & 5.01 & 1.76 \\
\hline
\end{tabular}

Diffraction angle and basal spacing of bebtonite and its modification with different organic cations suggested that $\mathrm{CH}$ and $\mathrm{AZ}$ have been intercalated into the Na-BNT galleries. The results were summarized in Table 1.

\section{FTIR spectroscopy}

FTIR is a useful technique to verify the presence of $\mathrm{CH}$ and $\mathrm{AZ}$ in the clay. Fig. 2 shows the FTIR spectra of Na-BNT, $\mathrm{CH}$ and $\mathrm{CH}-\mathrm{BNT}$ and Fig. 3 shows the FTIR spectra of Na-BNT, AZ and AZ-BNT. In Fig. 3, FTIR of the Na-BNT shows two peaks, which correspond to $\mathrm{Si}-\mathrm{O}$ stretching at $1047 \mathrm{~cm}^{-1}$ and interlayer water deformation vibration at $1645 \mathrm{~cm}^{-1}[17,18]$. The peak at $3628 \mathrm{~cm}^{-1}$ was from the $\mathrm{O}-\mathrm{H}$ stretching vibration. The peaks at $2850-2920 \mathrm{~cm}^{-1}$ and $2872-2939 \mathrm{~cm}^{-1}$

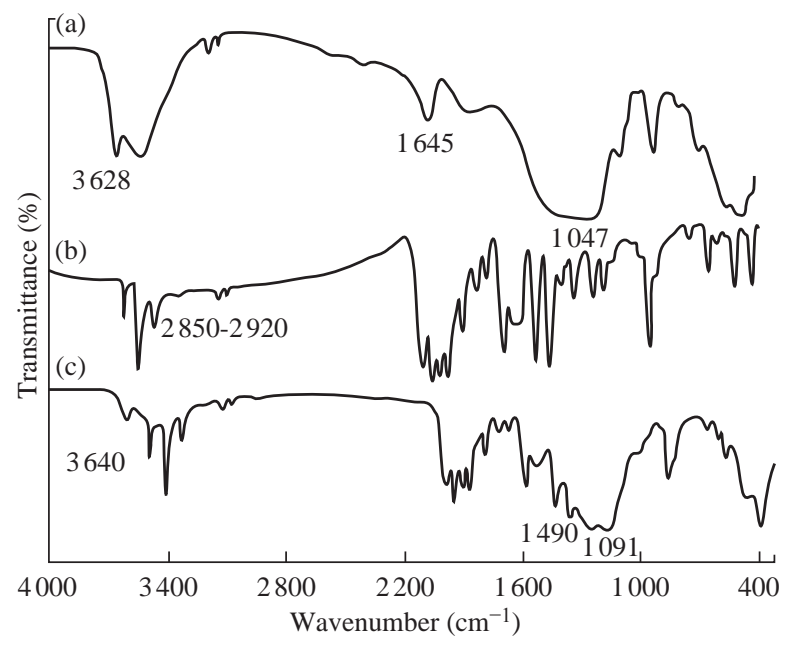

Fig. 2 FTIR Spectra of (a) Na-BNT; (b) CH; and (c) CH-BNT. 
correspond to the presence of the $\mathrm{C}-\mathrm{H}$ asymmetric and symmetric stretching vibration for $\mathrm{CH}-\mathrm{BNT}$ and AZ-BNT, respectively.

In the spectra of CH-BNT and AZ-BNT, the absorption bands at 3626 and $3640 \mathrm{~cm}^{-1}$ indicate the presence of free $\mathrm{O}-\mathrm{H}$ stretching and at 1091 and $1109 \mathrm{~cm}^{-1}$ correspound to $\mathrm{C}-\mathrm{N}$ stretching $[19,20]$. In addition to the bands of the original Na-BNT, the band at 1490 and $1438 \mathrm{~cm}^{-1}$ suggests the existence of the ammonium ion. Therefore, these results indicate that of $\mathrm{CH}$ and $\mathrm{AZ}$ were successfully intercalated in the silicate layers.

\section{NMR technique}

Fig. 4 shows ${ }^{1} \mathrm{H}-\mathrm{NMR}$ spectra of $\mathrm{CH}$ and $\mathrm{AZ}$, while ${ }^{13} \mathrm{C}$-NMR spectra of $\mathrm{CH}$ and $\mathrm{AZ}$ are shown in Fig. 5. ${ }^{1} \mathrm{H}-\mathrm{NMR}$ (300 MHz, DMSO) appearance of multiple signals at the site 6.3-7.8 ppm corresponded to the

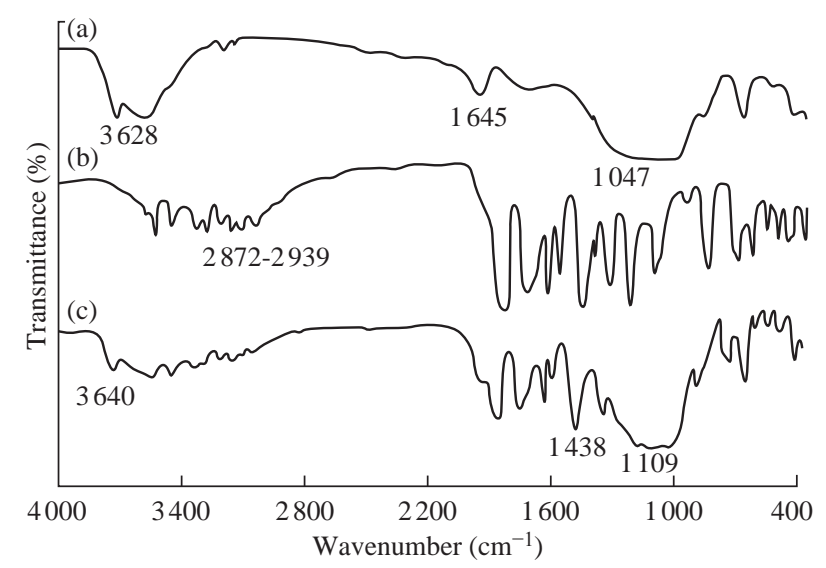

Fig. 3 FTIR Spectra of (a) Na-BNT; (b) AZ; and (c) AZ-BNT.
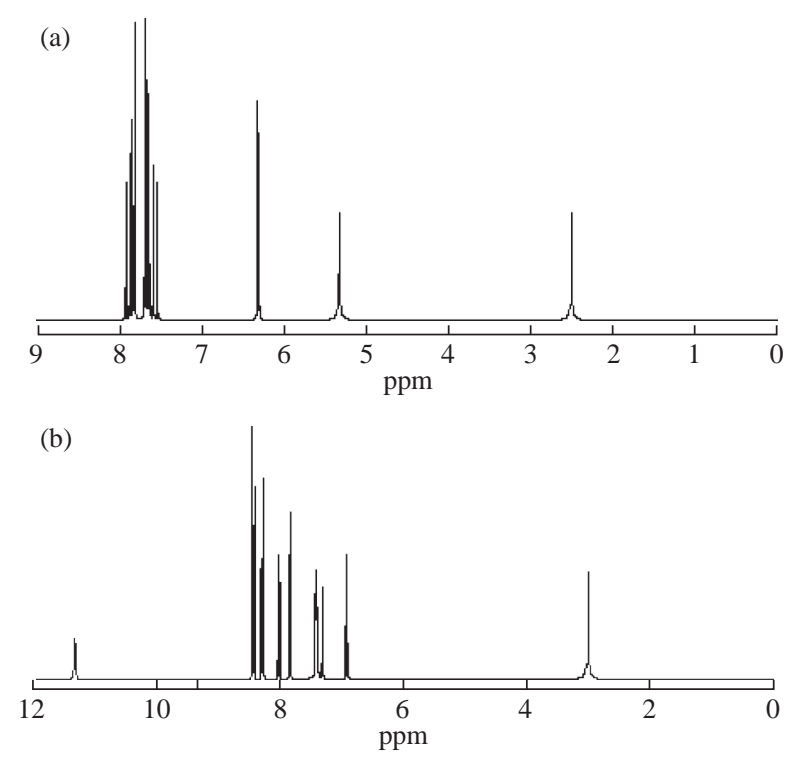

Fig. $4{ }^{1} \mathrm{H}-\mathrm{NMR}$ spectra of (a) CH; and (b) AZ.
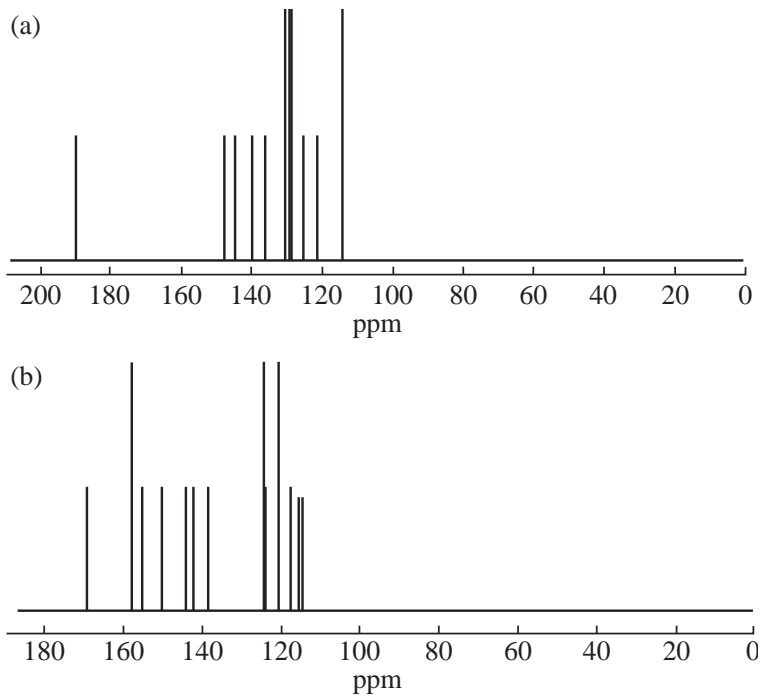

Fig. $5{ }^{13} \mathrm{C}$-NMR spectra of pure (a) pure $\mathrm{CH}$; and (b) pure AZ.

protons of benzene rings. The peak at the site $5.3 \mathrm{ppm}$ referred to the protons of $\mathrm{NH}_{2}$, and the appearance of signals at the site 7.5-7.9 ppm corresponded to the protons of $\mathrm{CH}=\mathrm{CH} .{ }^{1} \mathrm{H}-\mathrm{NMR}$ spectra revealed the appearance of multiple signals of AZ at the site 7.6-8.5 ppm, which was due to the protons of benzene rings. The peak at $6.5 \mathrm{ppm}$ corresponded to the protons of $\mathrm{NH}_{2}$, and the appearance of signals at the site 11-32 ppm was due to the protons of NH in sulfadiazine. The ${ }^{13} \mathrm{C}-\mathrm{NMR}$ (75 MHz, DMSO) (Fig. 3(a)) appearance of multiple signals at the site 114.0-130.6 ppm was due to the carbons of benzene rings. The peak at $189.7 \mathrm{ppm}$ referred to the carbons of $\mathrm{C}=\mathrm{O}$, and the appearances of signals at the site 145.2-121.3 ppm corresponded to the carbons of $\mathrm{CH}==\mathrm{CH}$. The peak at around $2.5 \mathrm{ppm}$ corresponded to DMSO as a solvent.

${ }^{13}$ C-NMR spectra of AZ (Fig. 3(b)) shows multiple signals at the site 114.3-169.3 ppm, which were due to the carbons of benzene rings.

\section{TGA analysis}

TGA graph shows the curves of weight loss for increasing temperatures of Na-BNT, CH-BNT and AZ-BNT (Fig. 6).

The TGA curves gave information on the structure of the intercalating molecules by the weight loss steps. Thermal degradation of BNT was shown to be before $200{ }^{\circ} \mathrm{C}$ because of the volatilization of water adsorbed on the external surfaces of the BNT and water inside the interlayer space. The BNT contained water due to the hydrated sodium $\mathrm{Na}^{+}$cations intercalated inside the clay layers. The presence of alkylammonium groups within the BNT interlayer spacing lowered the surface 


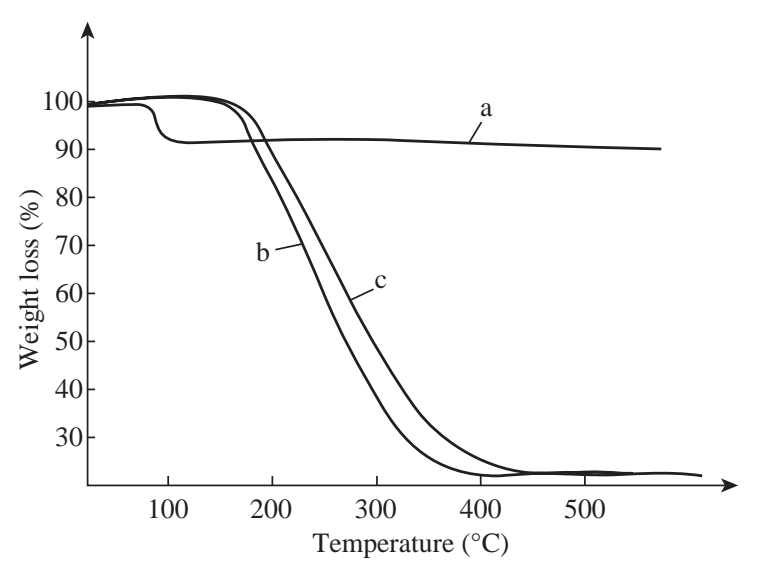

Fig. 6 TGA graph of (a) Na-BNT; (b) CH-BNT; and (c) AZBNT.

energy of the inorganic structure and would transform organophobic materials to organophilic materials ones.

The major difference between the thermogram of the unmodified clay and that of the organoclay was that the organic constituents in the organoclay decompose were in the range from 180 to $500{ }^{\circ} \mathrm{C}$.

\section{Conclusions}

Based on the results of this study, we concluded that the ammonium cations from $\mathrm{CH}$ and $\mathrm{AZ}$ ccould be successfully incorporated in the bentonite clay, and that the basal spacing of the bentonite clay increased as a result of incorporating $\mathrm{CH}$ and $\mathrm{AZ}$. We also found that the new organoclay could be susceptible to produce polymer nanocomposites, and that the thermal stability was enhanced by incorporation of clay into $\mathrm{CH}$ and AZ.

\section{References}

[1] S. Yun-Hwei, Preparations of organobentonite using nonionic surfactants. Chemosphere, 2001, 44: 989-995.

[2] A.M. Motawie, M.M. Madany, A.Z. El-Dakrory, et al., Physico-chemical characteristics of nano-organobentonite prepared using different organo-modifiers. Egy. J. Petroleum., 2014, 23: 331-338.

[3] W.F. Jaynes, S.A. Boyd, Clay mineral type and organic compound sorption by hexadecyltrimethyl ammonium exchanged clays. Soil. Sci. Soc. Am. J., 1991, 55: 43-48.

[4] J. Zhang, C.A. Wilkie, Preparation and flammability properties of polyethylene-clay nanocomposites. Polym. Degrad. Stability, 2003, 80: 163-169.

[5] M. Maiti, A.K. Bhowmick, Structure and properties of some novel fluorolastomer/clay nanocomposites with special reference to their interaction. J. Appl. Polym. Sci., 2005, 44: 162-176.
[6] E.A.J. Al-Mulla, Preparation of polylactic acid/epoxidized palm oil/fatty nitrogen compounds modified clay nanocomposites by melt blending. Polym. Sci - Series A., 2011, 53: 149-157.

[7] M.A. Motawie, N.M. Ahmed, S.M. ElMesallamy, et al., Unsaturated polyesters/layered silicate nanocomposites: Synthesis and characterization. IOSR J. of Appl. Chem., 2014, 7(10): 34-43.

[8] S.M.M. Al-Mutoki, B.A.K. Al-Ghzawi, and A.A. Abdullah, et al., AIR AlAmmar Synthesis and characterization of new epoxy/titanium dioxide nanocomposite. Nano Biomed. Eng., 7 (4): 135-138.

[9] S.A. Sadiq, E.M. Atiyah, and A.T. Numan, Synthesis and characterization of new bidentatechalcone ligand type (NO) and its MnII, CoII, NiII and CuII complexes with study of their antibacterial activity. Diyala J. Pure Sci., 2015, 11(3): 2222-2235.

[10] F.W. Salman, S.W. Radhi, and A.M. Jodh, Preparation and study antibacterial activity of a new polymer. J. of Babylon Pure Appl. Sci., 2015, 2: 23-31.

[11] E.A.J. Al-Mulla, A.H. Suhail, and A.A. Saadon, New biopolymer nanocomposites based on epoxidized soybean oil plasticizedpoly(lactic acid)/fatty nitrogen compounds modified clay: Preparation and characterization. Ind. Crops Prod.., 2011, 33: 23-29.

[12] E.A.J. Al-Mulla, W.M.Z. Yunus, N.A. Ibrahim, et al., Enzymatic synthesis of fatty amides from palm olein. $J$. Oleo. Sci., 2010, 59(2): 157-160.

[13] E.A.J. Al-Mulla, W.M.Z. Yunus, and N.A. Ibrahim, Epoxidizedpalmoil plasticized polylactic acid/fatty nitrogen compound modifiedclaynanocomposites: Preparation and characterization. J. Polym. Comp., 2010, 18(8): 451-460.

[14] W.M.Z. Yunus, N.A.B. Ibrahim, and M.Z.A. Rahman, Difatty acyl urea from corn oil: Synthesis and characterization. J. Oleo Sci., 2010, 59: 157-160.

[15] Y.J. Phua, W.S. Chow, and Z.A. MohdIshak, Organomodification of montmorillonite and its effects on the properties of poly(butylene succinate) nanocomposites. Polym. Eng. Sci., 2013, 53: 1947-1957.

[16] B.A. Mansor, H.H. Wisam, N.I. Azowa, Modification of montmorillonite by new surfactants. J. of .Eng. Appl. Sci., 2009, 4(3): 184-188.

[17] F.H.J. Al-Shemmari, E.A.J. Al-Mulla, and A.A. Rabah, A comparative study of different surfactants for natural rubber clay nanocomposite preparation. Rendiconti Lincei. 25(3): 409-413.

[18] L. Guo, S. Wu, and F. Zeng, Synthesis and Fluorescence property of terbium complex with novel Schiff-base macromolecular legend. Eur. Polymer J., 2006, 42: 16701675.

[19] E. Ramachandranl, K. Baskaran, and S. Natarajan, XRD, thermal, FTIR and SEM studies on gel grown gammaglycine crystals. Crys. Res. Tec., 2007, 42: 73-77.

[20] S.M.M. Al-Mutoki, A.G. Wadday, and A.A. Abdullah, Effect of nano $\mathrm{TiO}_{2}$ dopant on electrical properties of SR8100/nano $\mathrm{TiO}_{2}$ PMNC. Results Phys., 2016, 6: 551553.

Copyright $(2017$ Manar Ghyath Abd-Almutalib Al-Mosawy, Emad Abbas Jaffar Al-Mulla, and Majed Jari Mohamad. This is an open-access article distributed under the terms of the Creative Commons Attribution License, which permits unrestricted use, distribution, and reproduction in any medium, provided the original author and source are credited. 